\title{
ON THE CAUSES
}

\section{OF THE \\ ENDEMIC PUERPERAL FEVER OF VIENNA.}

By C. H. F. ROUTH, M.D.,

Communicated by Dr. MURPhY.

Received May 29th.-Read Nov. 28th, 1848.

TuE author of the following remarks having had his attention painfully called to two or three unfortunate cases of puerperal fever, prior to his visit to the Continent, had subsequently an opportunity, in walking the hospitals of Paris and Vienna, of obtaining some more extended information relative to the causes of this disease. Believing that the facts he has observed are sufficiently important, he ventures to call to them the attention of the profession. These remarks, however, must be regarded as applying especially to Vienna, although it is believed that similar causes are brought into operation in other countries, and might as effectively be combated.

A short explanation of the manner in which labours are conducted in the Vienna Hospital, and the system of clinical instruction carried on, is a necessary prelude to a clear understanding of the question.

There are three lying-in departments in the general hospital. The first, a secret department, to which strangers are not admitted, and which, therefore requires no further comment. The remaining two are open to medical investigation. For the sake of clearness, we shall speak of one of these as the first division, and of the other as the seconddivision.

The first division is for the instruction of medical men and midwives. The second division for midwives exclusively. 
In the former there are about twenty or thirty medical men, and eight midwives. These midwives, in addition, perform other menial duties. There are besides about sixteen nurses employed in the various wards to attend upon the patients. The wards in this division are eight in number: they are large and airy.

In the second division, or that exclusively intended for midwives, their number is about twenty-eight, but in other respects the arrangements are entirely similar to those of the first division, with this exception, however, which it is important to bear in mind, that the wards are neither so large nor so airy.

The following table exhibits the average number of deliveries and the amount of mortality per month :

\begin{tabular}{|c|c|c|c|c|c|c|c|c|}
\hline \multirow{3}{*}{$\begin{array}{l}\text { Number of } \\
\text { deliveries in } \\
\text { each division. }\end{array}$} & \multicolumn{4}{|c|}{ First Division-Mortality. } & \multicolumn{4}{|c|}{ Second Division-Mortality. } \\
\hline & \multicolumn{2}{|c|}{ Ordinary. } & \multicolumn{2}{|c|}{ Occasional. } & \multicolumn{2}{|c|}{ Ordinary. } & \multicolumn{2}{|c|}{ Occasional. } \\
\hline & $\begin{array}{c}\text { Actual } \\
\text { number } \\
\text { of deaths. }\end{array}$ & $\begin{array}{c}\text { Propor- } \\
\text { tiontode- } \\
\text { liveries. }\end{array}$ & $\begin{array}{c}\text { Actual } \\
\text { number } \\
\text { of deaths. }\end{array}$ & $\begin{array}{l}\text { Propor- } \\
\text { tiontode } \\
\text { liveries. }\end{array}$ & $\begin{array}{c}\text { Actual } \\
\text { number } \\
\text { of deaths. }\end{array}$ & $\begin{array}{l}\text { Propor- } \\
\text { tion tod } \\
\text { liveries. }\end{array}$ & 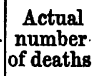 & $\begin{array}{l}\text { Propor- } \\
\text { tion tode- } \\
\text { liveries. }\end{array}$ \\
\hline $\mid \begin{array}{c}\text { From } \\
250-300 \\
\text { mean } 275\end{array}$ & $\begin{array}{c}\text { about } \\
30\end{array}$ & $\begin{array}{l}1 \text { in } \\
9 \cdot 16\end{array}$ & $\begin{array}{c}\text { about } \\
70\end{array}$ & $\begin{array}{l}1 \text { in } \\
3 \cdot 92\end{array}$ & $\begin{array}{l}\text { about } \\
7 \text { to } 9 \\
\text { mean } 8\end{array}$ & $\begin{array}{c}1 \text { in } \\
34 \cdot 37\end{array}$ & $\begin{array}{c}\text { about } \\
3\end{array}$ & $\begin{array}{c}1 \text { in } \\
91 \cdot 66\end{array}$ \\
\hline
\end{tabular}

In one particular year, indeed, out of some 3000 cases delivered, about 500 died in the first division. While in the second division, out of the same number delivered, the deaths frequently did not exceed forty. These numbers are only approximative, as for obvious reasons the statistics are kept secret.

The clinical instruction is conducted on this wise. In the first division the management of labours is thus regulated : four of the midwives are reserved for the night duty, and four for the day duty, and each of these must, in her turn, deliver a woman; but one is moreover called upon every eight days to act as the "Journalistin," i. e. to watch and examine every case in labour admitted during a period of twenty-four hours. Two gentlemen (sometimes only one) are called upon to exercise, conjointly with the midwife in 
question, a similar function. In the discharge of this duty, they are allowed to repeat these examinations as frequently as they deem it necessary. But this is not all. When the membranes are ruptured, the midwife, according to order, is called to deliver the woman, and one gentleman (formerly there were two) is also in attendance to witness the delivery. This is what is called "taking a tour," and these parties are again allowed to make examinations, if they think fit. Again, the professor during his morning visit, and the assistant during the evening visit, not unfrequently make or allow examinations to be made by the gentlemen present, when. ever a case of interest presents itself. Practically, therefore, every woman admitted must needs be examined by some five different persons at least, and this number may be doubled or even trebled. The practical instruction is also given in a private course, where the operations are performed upon the dead body of some female.

The clinical instruction in the second division is conducted on precisely the same general plan, and the examinations made are as frequent, if not more so; but the practical instruction is given and the operations performed upon the leather phantom, and not upon the dead body. This last fact it is important to remember. These details may appear somewhat tedious, but they bear too much upon the sequel to have been omitted.

The frightful mortality in the first division, as compared with the second division, became at last so notorious, that many females refused to become patients in the former, and public opinion compelled the Austrian government to make it a subject of inquiry. The commission appointed for that purpose came to the conclusion that the prevalence of the fever was due to the excess of students in attendance, and their number in consequence was reduced from forty to about thirty. The same disproportion in the mortality of the two divisions, however, still continued, so that it was evident to all that the true cause had not been discovered.

This singular difference between the two divisions could not fail to occupy a large share of the attention of the pro- 
fession, the more so, as analogous examples were traced, or were supposed to have been found, in other countries. 'Thus in Prague, where two divisions also existed, it was said that the mortality was even greater than in Vienna, in the division for medical men; while in that for midwives exclusively, it was but small. In Strasburg, likewise, the author was credibly informed by Dr. Wrieger, a practitioner of that city, where there are also two divisions, that the same difference of mortality was observed, so much so, as to make it sometimes imperative to close the division for medical men, for the purpose of disinfecting the wards; while in that for midwives puerperal fever was very rare. The proverbial mortality in the "Hôpital Clinique" of Paris, where medical men and students only are in attendance, was supposed to afford melancholy proof of the baneful results consequent on the attendance of male obstetricians. The first and most natural presumption seemed to be that the fever was excited in the first instance by the rough manipulations of men-midwives, these setting up inflammation about the generative organs, which, by extension upwards, gave rise to puerperal fever; while the manipulations of the female hand being more gentle, no such unfortunate complication followed in the cases they attended. A very little reflection and a positive example to the contrary at once disproved this supposition.

1st. As Dr. Semelweiss (to whom I shall more particularly allude immediately) remarks, if it were so, it is evident that, in all cases of difficult or instrumental labour, puerperal fever would result, whereas this is by no means the general rule.

2d. Male obstetricians are, for the most part, persons whose anatomical and physiological knowledge of the parts concerned in labour exceeds that of midwives, and to whom therefore a diagnosis, by being so much more easily and speedily made, does not necessarily require so much manipulation, so that one of the chief causes of inflammation, and consequently of puerperal fever, is thus avoided.

$3 \mathrm{~d}$. This fever is comparatively rare in the private practice of accoucheurs. 
4th and lastly. The positive example to the contrary is afforded us by the Hôpital de la Maternité, at Paris, where the ordinary labours are exclusively conducted by midwives, and where the disease is of very frequent occurrence.

We must, therefore, conclude that there is no evidence to show that the roughness of the manipulations of male obstetricians is the cause of puerperal fever.

Contagion.-At a period when the occurrence of so many diseases is ascribed to this influence, it is proper to consider how far it may be capable of exciting puerperal fever. That the contact of fomites, the direct or indirect communication with persons affected with puerperal fever; that some influence carried, either by the patient herself or her attendants, from other contagious diseases, such as erysipelas, typhus, and other low fevers, has in many cases given rise to puerperal fever among lying-in patients, is a fact that admits of no doubt. Drs. Copland, Collins, Rigby, Lee, Gooch, Ferguson, Blackmore, Piddie, Mr. Storr, and a host of other writers, have related several cases of this kind. Again, Dr. E. Murphy, of London, has especially called attention to the late epidemic of Dublin, in which it was clearly proved, that when the medical man in attendance on a puerperal fever case visited another female in labour, and delivered her in the same clothes in which he had attended the first, he almost invariably communicated the fever to her. This fact was peculiarly exemplified in the case of a German student, who was following the midwifery practice in and out of the hospital, and being rather neglectful in his habits, and not changing his clothes as desired, invariably communicated the fever to all the cases he attended, whereas with others, who were more careful in this respect, no such unfavorable results occurred.

The author was aware of these facts, and made particular inquiries on the subject in Vienna; but whether it was due to the peculiar variety of the Vienna fever or not, the information he obtained was altogether nugatory in regard to this view. At first, indeed, it did appear probable that it might be induced by the contagion of typhus, which is known 
to be one of the most common and fatal diseases in Vienna. He could not, however, hear of a single case of puerperal fever that could be traced satisfactorily to this contagion, nor conversely of a single case of typhus occurring in any of the nurses or attendants of the cases affected with puerperal fever.

2d. As to its propagation more particularly by the clothes of the attendants-the very method of tuition in Vienna disproves this. The medical men and midwives in the first division are continually by the bedside of the puerperal fever cases, and generally in the same dress. Indeed the author, amongst many others, kept a particular dress for doing duty in that hospital, and he never had reason to believe that he had been the medium of contagion. Moreover, he has negative evidence to the contrary, i. e. of having frequently made a careful medical examination of a patient affected with most malignant puerperal fever, and remained by the bedside for several minutes together, and subsequently examined some ordinary case in the ward, and yet the disease was not communicated. In like manner, he has frequently remarked that the patients on each side a puerperal fever case have entirely escaped the disease. Such facts he is quite aware cannot be regarded as very positive evidence; but negative evidence frequently repeated, assumes a certain positive value; and it is here strengthened by the facts observed in the second division. As it has already been stated, the same regulations are enforced in the second division, and the same favorable conditions for contagion are present, with this difference only, that partly from these regulations being more stringent, and partly from the very habits of women, the midwives are more frequently and longer at the bedside. Were contagion then the cause, we should expect that puerperal fever would be of more frequent occurrence in the second than in the first division, which is the reverse of the fact.

3dly. The Strasburg Hospital seems to disprove the contagious nature of the disease. Here it has generally been observed (and that usually every two years), that the 
fever becomes so pestilential, as to necessitate the closure of the establishment for the purpose of disinfecting the wards. The females who at the time may be affected with puerperal fever are then removed to the division for midwives. Experience has shown that in such instances the disease does not spread. This statement is made upon the authority of Dr. Wrieger, of Strasburg.

4thly. Allusion has been already made to the eight midwives and sixteen nurses, in the first division at Vienna. Now, the former must, by law, be married women; and it is believed the same rule applies to the nurses. At any rate, it frequently happens that they become pregnant. Here we have persons in constant attendance upon the puerperal fever cases. Most, if not all, of these are usually confined in the hospital ; the only difference in their case being, that they are generally delivered by one of the head-nurses, apart in a private ward, and that the medical men do not assist in the confinement. Now, although we have the means of contagion present here in an eminent degree, the author was, on careful inquiry, unable to hear of a single case where one of the nurses or midwives had puerperal fever.

5thly and lastly. The only fact which would appear to give some countenance to the view of contagion is the following: It often occurred that the infant of the female affected with puerperal fever died at the outset of the mother's illness; and, in such cases, a post-mortem examination of the infant proved death invariably to have resulted from peritonitis. The fact is important, when taken in connexion with the occurrence of peritonitis in husbands exposed to the contagion of puerperal fever from their wives, as noticed by Mr. Storr. But here the evidence is not sufficiently clear to allow us to attribute the result to mere contagion. As there are good grounds for believing that the lochia in ordinary puerperal fever is intensely poisonous in most cases, as also puerperal abscesses, \&c., and that there is every reason to believe that the entire mass of the blood is poisoned in such cases; that, on the other hand, the child is very easily affected by any morbid qualities of the mother's 
milk (which, of all the secretions, is, perhaps, that most easily modified by the state of the mother), it seems more correct to conclude that the milk in this case becomes an animal poison to the child, the peculiar effect of which (in the same manner as cantharides may produce an inflammation of the urinary passages) is to produce peritonitis. It is not thought, therefore, that, in the facts adduced, there is sufficient evidence to conclude that, either by the contact of fomites, or communication of the sick with infected persons, directly or indirectly, the Vienna puerperal fever is contagious.

Infection.-Infection properly so called, that " peculiar morbid atmospheric influence which extends beyond the range of personal communication," through the respiratory organs perhaps, and which is distinct from epidemic influences, as well as from true contagion,-this influence, the author does not believe, could be unequivocally made out to exist in the Vienna Hospital. Many of the facts already adduced in speaking of contagion go far to disprove infection also; such as the non-occurrence of puerperal fever among the nurses. Moreover, the circumstances known to favour infection, i. e. want of cleanliness and ventilation in the wards, are absent. The patients are delivered in a ward especially set aside for that purpose. Moreover, on removal clean sheets are always given. The lying-in chamber is partly cleaned every morning, thoroughly every three or four days, and even the very straw mattresses are changed; and so it is with every ward. It may reasonably therefore be said that the greatest cleanliness prevails throughout the department; and generally the moment a patient becomes really sick with malignant puerperal fever, she is at once removed into a separate apartment. But, secondly, we have a strong argument against infection exerting so powerful an influence, in this fact, that if these regulations were inefficient, the disease ought to be infinitely more frequent and fatal in the division for midwives, where the wards are altogether less roomy and less airy. These considerations, it is believed, warrant the conclusion, that infection cannot be rightly 
looked upon as the cause.which originates the puerperal fever of Vienna.

Direct application of poisonous matter, or inoculation.Dr. Blackman, of Edinburgh, it is believed, was the first who distinctly stated that it was probable the medical attendant might communicate the disease, by retaining a portion of poison, subter ungues, which he subsequently directly applied on making an examination per vaginam.* Though this fact may have been suspected by many others, yet, so far as the author is aware, he believes that it was first clearly enunciated by Dr. Semelweiss, the distinguished assistantphysician of the first obstetric division at Vienna. Reasoning upon some of the facts before noticed, Dr. Semelweiss was led to infer that the real source of the infection was to be found in the hands of the medical men in attendance contaminated with cadaveric poisons. These gentlemen having all more or less diligently attended the numerous autopsies in the dead-house, and frequently handled, as a matter of course, the specimens of disease found. Now, Dr. Semelweiss remarks that, after handling dead matter, the hands, however well washed, still retain a peculiarly fetid cadaveric odour, which does not disappear for several hours, and sometimes not till the next day. The very presence of this smell implies the retention on the surface, or imbibition by the epidermis of a quantity, however small, of cadaveric matter. Again, when it is remembered that in many dead-houses, from the absence of a nail-brush, frequently of soap and warm water, the hands cannot be properly washed, it is exceedingly probable that a portion of this cadaveric matter is retained under the nails ; and it is very easy to understand how, on examination made subsequently per vaginam, this poison should be directly and effectively applied. In this manner he supposed the disease was so frequently generated in the wards of the first division, and explained the sanitary condition of the second division, since the midwives made no autopsies, and worked on the phantom, and so did not get their hands infected with cadaveric matter.

* Prov. Med. and Surg. Journal, No. xxv, 1845. 
But Dr. Semelweiss did more. He acted upon this supposition. He recommended all students frequenting the division not to handle dead matter, or if they did, he forbad them to make any examination till the following day. In the second place, he directed all the students who attended the practice of the division to wash their hands in a solution of chlorine prior to and after every examination made on the living subject. The result of these precautionary measures was that the number of deaths at once fell to seven per month, or the usual average of the second division. This is a speaking fact. It explains the difference of mortality of the two divisions at Strasburg. Here, likewise, although the medical men in attendance do not dissect, yet they perform operations upon the dead body and autopsies, whereas the midwives do not. The difference of the mortality at Prague may also be so accounted for. The apparent exception of the Maternité at Paris is also explained, since the midwives in that institution do dissect and perform the autopsies; and with regard to the Hôpital Clinque at Paris, the known zeal of the French students for anatomical inquiries, coupled with their general indifference in the selection of bodies for dissection, will also fully account for the frightful frequency of puerperal fever in that hospital.

Even in Great Britain many of the cases reported and ascribed to contagion from typhus or erysipelas, may in reality have been caused by the direct inoculation of poisonous secretions derived from gangrenous wounds in such cases, with which the hands of the medical attendants, who had previously dressed these wounds, had been accidentally infected.* This view of the case derives some additional confirmation, from the similarity or analogy of the symptoms of puerperal fever with those arising from a poisoned dissection wound.

This poisonous matter may be absorbed in several ways. Through the absorption of the mucous membrane of the vagina, or more directly through trivial wounds about the

* Dr. Peddie's case, Prov. Med. and Surg. Journal, No. xx, 1846 ; Mr. Storr's case, ibid., No. clxvi, 1843. 
generative organs, such as the lacerated fourchette in primiparæ, lacerations of the cervix or os uteri, or the extended inner surface of the uterus deprived of its decidua and placenta; all these parts, both from the general nervous shock and the removal of local congestion, being in a highly favorable condition for absorption.

The feverish symptoms usually occurred on the second or third day after labour; yet cases were noticed where they did not occur before the seventh and even fourteenth days. Generally, the fatal result did not take place before the fourth or fifth day after seizure, but sometimes it occurred as early as twenty-four hours after the first appearance of the fever. A diarrhœa generally preceded the attack, but sometimes, though rarely, the patient was costive. Then followed general languor, and a feeling of weakness, rigors; and the pulse was usually from 120 to 130 in the minute, sometimes, however, as high as 160 , very weak. The patient's countenance assumed a peculiarly anxious expression; there was pain generally about the uterus, either in the organ itself or in the peritoneum about it. As these symptoms increased in intensity, heat set in, with dry tongue, loss of appetite, thirst, \&c. The lochia was very seldom affected either in quantity or appearance. The lacteal secretion continued undiminished, and apparently unaltered. Indeed, if at the outset of the disease the skin was observed to be rather drier than usual, this symptom did not last; the perspiration becoming subsequently more copious than otherwise, and continuing so up to the hour of dissolution. Large violet maculæ occurred sometimes in the extremities. In some cases the intellect was affected, but this was of very rare occurrence, as it usually continued clear up to a very few minutes before death. The more marked local symptoms were those of peritonitis, which gradually increased in intensity. The pain generally disappeared an hour before death; the face gradually assuming a more sallowish, yellow, and cadaveric expression.

The post-mortem appearances were generally the following :-Arachnitis in a slight degree. Occasionally endocar- 
ditis, very generally pericarditis. Almost always some pleuritis or pneumonia. Liver and spleen healthy. Extensive peritonitis, with plastic adhesions, and sero-purulent fluid in the abdominal cavity. The uterus was much enlarged, much softened, and tearing very readily, containing pus in the veins, especially in the neighbourhood of the fallopian tubes. The inner surface was of a yellow-whitish colour, with here and there plastic exudation. Sometimes the lymphatics proceeding along the spine were beaded in appearance, containing pus. Where death occurred early, there was generally only some muddy-looking fluid in the abdominal cavity, with endometritis, and occasionally pus in the uterine veins. Sometimes the case was one of pure peritonitis, the uterus being scarcely affected. The body was very prone to decomposition ; its external surface mottled, especially at the extremities; the superficial veins very apparent, of a dull bluish-red colour. The blood generally liquid, and very dark, not coagulating after exposure.

The fever was not equally intense in all cases. This might be due to the idiosyncrasy of the female, or to the variety of the poison. Indeed, Dr. Semelweiss thought he had observed that the inoculation of cadaveric matter from cases of typhus, erysipelas, and puerperal fever was most fatal.

The author, therefore (although he is perfectly willing to admit that the fever, like all other fevers, may be very much modified by epidemic influences), thinks himself justified in concluding -

In the first place, that-1. The puerperal fever of Vienna may be looked upon as an endemic fever.

2. That it is produced by the direct application of poisonous cadaveric matters, introduced by the hands of the male obstetricians in attendance.

3. That the cadaveric matters derived from the bodies of persons dead of typhus, erysipelas, puerperal or other low fevers, are peculiarly dangerous; but that there is no evidence to show that any ordinary cadaveric matters may not produce it.

4. That it does not appear to be either contagious or infectious. 
And, in the second place, as the precautionary measures adopted by Dr. Semelweiss have produced such satisfactory results, that-

1. Accoucheurs should as much as possible avoid making autopsies, and all contact with cadaveric matter.

2. They should never attend any woman in labour in clothes which may possibly have been infected; but, especially if compelled to handle cadaveric matters, or other poisonous secretions from living persons, should, prior to any examination made per vaginam, not be satisfied with washing their hands most carefully, and subsequently greasing them, but also make use of a solution of chlorine, to disinfect the hand from any poisonous matters with which it may have become contaminated, before they presume to enter the lying-in bedchamber.

Postscript : April 29, 1849.-Accounts which reached me through Dr. Tumanhof, in June last, or six months after my departure from Vienna, confirmed in every respect the favorable results obtained from the adoption of Dr. Semelweiss's precautionary measures. And in a letter since received from Dr. Golling, Secundarius to the hospital, bearing date December 24th, 1848, the following passage occurs: "Dr. Semelweiss and all the medical men of the hospital are delighted with the admirable success of his system." The origin of the Vienna puerperal fever seems therefore to be fully established.

May 21, 1849. - In a letter received from Dr. Semelweiss a few days since, dated March 4th, 1849, the following statistics are given: In one division (the first division)-

Females.

In 1846 there were admitted 3354 and there died 459 
It was not till the end of May, 1847, however, that the chlorinated lotions were employed. Thus, you see that the mortality has diminished in an extraordinary manner.

Professor Michaelis, of Kiel (Schleswig-Holstein), writes me that, from the time he has employed chlorinated lotions, which extends over a space of several months, he has only lost one female; though, prior to that period, the mortality was so great, that he was considering whether it would not be right to close the hospital altogether. 